\title{
Lo nuevo en diagnóstico y tratamiento de diabetes mellitus gestacional
}

New evidence on diagnosis and treatment of gestacional diabetes mellitus

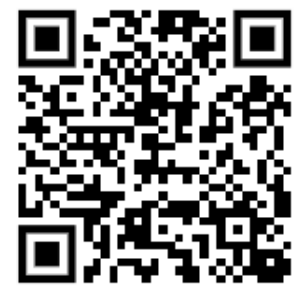

'Dra. Andrea Espinoza Artavia

Investigadora independiente, San José, Costa Rica a.espinozaartavia@gmail.com

https://orcid.org/0000-0001-5512-2177

${ }^{2}$ Dra. Roxana Fernández Vaglio Investigadora independiente, San José, Costa Rica rox.fernandez.vaglio@hotmail.com

https://orcid.org/0000-0003-2780-7560

RECIBIDO

$14 / 02 / 2019$
CORREGIDO

$09 / 03 / 2019$
ACEPTADO

$11 / 03 / 2019$

\section{RESUMEN}

La diabetes mellitus gestacional es una condición en la que se desarrolla intolerancia a los carbohidratos y se diagnostica por primera vez en el embarazo sin una pre-existencia clara de diabetes tipo 10 tipo 2. Es una patología de gran importancia debido a su creciente prevalencia y asociación a complicaciones maternas, fetales y/o neonatales que afectan a múltiples áreas de la medicina y la comunidad. Pese a que aún hay controversia respecto a su diagnóstico, múltiples estudios recomiendan el método de 2 pasos basado en un tamizaje con 50 gramos de glucosa oral y posteriormente una prueba de tolerancia de glucosa de 3 horas con una ingesta de 100 gramos de glucosa en aquellas que presenten un tamizaje positivo. El pilar del tratamiento va a consistir en la dieta y estilos de vida saludable. Para aquellas pacientes que requieran tratamiento farmacológico, el principal ente va a seguir siendo la

${ }^{1}$ Médico general, graduada de la Universidad de Costa Rica (UCR), investigadora independiente, San José, Costa Rica. Código médico: 15174.

${ }^{2}$ Médico general, graduada de la Universidad de Costa Rica (UCR), investigadora independiente, San José, Costa Rica. Código médico: 15160. insulina, pudiendo utilizar asimismo la metformina como una alternativa viable. Aún queda mucho por explorar y determinar en esta patología compleja, para lograr alcanzar un consenso entre las distintas guías y conseguir las mejores recomendaciones para la población a escala mundial.

PALABRAS CLAVE: embarazo; diabetes gestacional; estilo de vida: insulina; glucosa.

Gestational diabetes mellitus is a condition in which carbohydrate intolerance develops and is diagnosed for the first time in pregnancy 
without a clear pre-existence of type 1 or type 2 diabetes. It is a pathology of great importance due to its increasing prevalence and association to maternal, fetal and/or neonatal complications that affect multiple areas of medicine and the community. Although there is still controversy regarding its diagnosis, multiple studies recommend a 2step method based on a 50-gram oral glucose screen and then a 3hour glucose tolerance test with a 100-gram glucose ingestion in those women that present a positive screening. The mainstay of the treatment will consist of healthy diet and positive lifestyles. For those patients that require pharmacological treatment, the main entity will continue to be insulin, though one may also use metformin as a viable alternative. There is still much to explore and determine in this complex pathology, in order to achieve consensus between the different guidelines and obtain the best recommendations for the population worldwide.

KEYWORDS: pregnancy; diabetes; gestational; life style; insulin; glucose.

\section{INTRODUCCIÓN}

La diabetes mellitus gestacional (DMG) es una condición en la que se desarrolla intolerancia a los carbohidratos, y se diagnostica por primera vez en el embarazo sin una pre-existencia clara de diabetes tipo 1 o tipo 2 (1). Es de las complicaciones médicas más comunes del embarazo, afectando tanto a la madre como al feto (o neonato) a corto, mediano y largo plazo. Sin embargo, pese a múltiples estudios a través de los años y décadas de investigación, aún hay debate entre los expertos respecto a su correcto manejo. Este artículo de revisión se realiza con el propósito de establecer una guía rápida de diagnóstico y manejo de la diabetes mellitus gestacional basada en bibliografía reconocida y reciente, además de identificar las posibles brechas que aún existen para futuras investigaciones respecto al tema.

\section{MATERIALES Y MÉTODOS}

Para la elaboración de esta revisión, se utilizó bibliografía reciente publicada en revistas mundialmente reconocidas como la ADA, FIGO y ACOG, enfocándose en el tema de diagnóstico y tratamiento de diabetes mellitus gestacional exclusivamente. Se tomó en cuenta el uso de bibliografía tanto en inglés como en español.

\section{DIAGNÓSTICO}

\section{- GENERALIDADES}

La prevalencia de la DMG varía en directa proporción a la prevalencia de diabetes tipo 2 en una población, raza o etnia. Por ejemplo, las mujeres caucásicas generalmente tienen los menores índices de DMG, y por el contrario, las mujeres hispánicas, afroamericanas, amerindias y asiáticas o isleñas del Pacífico tienden a tener una 
alta prevalencia de DMG (2).

El riesgo de padecer DMG va a incrementarse con los mismos factores de riesgo establecidos para la diabetes tipo 2, tales como: obesidad, edad avanzada, sedentarismo, entre otros. Al existir una mayor prevalencia actual de estilos de vida poco saludables, la prevalencia de DMG en mujeres de edad reproductiva ha aumentado considerablemente $(1,3)$. Asimismo, al mismo tiempo que la edad para el inicio de diabetes está disminuyendo considerablemente, en muchas poblaciones la edad para el embarazo ha aumentado, por lo que muchas mujeres son vulnerables a entrar en estado de hiperglicemia (4).

Hace muchos años, el tamizaje para DMG consistía únicamente en obtener la historia médica de cada paciente, refiriéndose a eventos obstétricos en embarazos previos y a antecedentes histórico-familiares de diabetes tipo 2. A partir de 1973, un estudio propuso el uso de una ingesta de 50 gramos de glucosa para realizar una prueba de tolerancia de 1 hora como herramienta de tamizaje para DMG, la cual ha sido ampliamente utilizada desde entonces (5).

Se sabe que algunas mujeres poseen bajo riesgo de padecer de DMG, por lo que no sería costo-efectivo realizar el tamizaje en esta población. Sin embargo, esto constituye aproximadamente solo el $10 \%$ de mujeres embarazadas, por lo que identificarlas y excluirlas podría constituir una complejidad innecesaria para el proceso (2). Además, se ha demostrado que el uso de antecedentes familiares o personales de diabetes, eventos adversos en embarazos previos, glucosuria y obesidad para identificar la DMG fallará en identificar al menos la mitad de embarazos con DMG. Debido a esto, a partir del 2014 el Grupo de Trabajo de Servicios Preventivos de los Estados Unidos (U.S Preventive Services Task Force en inglés) recomienda realizar el tamizaje en todas las mujeres embarazadas para DMG a las 24-28 semanas de gestación $(6,7)$.

\section{- DIAGNÓSTICO DE DOS PASOS}

En el 2013, el Instituto Nacional de la Salud (NIH por sus siglas en inglés) realizó una conferencia para llegar a un consenso respecto a los criterios diagnósticos para la DMG. En el mismo, se llegó al acuerdo de seguir el protocolo de 2 pasos, que hoy en día es el recomendado por el Colegio Americano de Obstetras y Ginecólogos (ACOG, por sus siglas en inglés) (1). El primer paso, como se explicó previamente, consiste en el tamizaje con la ingesta de 50 gramos de una solución glucosada con una determinación de glicemia una hora posterior a esto. Para las mujeres que alcanzan o exceden el valor establecido para el tamizaje, se deberá de realizar un segundo paso que consiste en una ingesta de 100 gramos de glucosa con una determinación de glicemias a lo largo de 3 horas. Tradicionalmente, el diagnóstico definitivo se realiza cuando se exceden al menos 2 de los 4 valores esperados para la curva de tolerancia de glucosa (2).

Existen datos insuficientes para determinar si el valor de corte para el tamizaje de la DMG debería de establecerse en algún punto específico entre 130-140 mg/dL, basado en efectividad o mejor desenlace de eventos obstétricos adversos. Un estudio de cohorte demostró que al establecer el 
corte en $140 \mathrm{mg} / \mathrm{dL}$ disminuye la cantidad de falsos positivos y mejoran los valores predictivos positivos en varios grupos étnicos y raciales, además de evitar la administración innecesaria de la prueba diagnóstica, la cual se ha visto relacionada con estrés e insatisfacción materna con respecto al proceso. Asimismo, demostró que la sensibilidad del estudio mejoraba sólo marginalmente al utilizar valores de corte inferiores. Pese a que se ha recomendado la estandarización de un valor, en ausencia de evidencia clara, se ha propuesto que cada profesional seleccione un valor consistente para su práctica clínica, con base en factores y prevalencias propias de cada comunidad (2).

Se han descrito diferentes cortes para las mediciones de la curva de tolerancia de glucosa. El Grupo de Datos Nacional de Diabetes (National Diabetes Data Group) establece los cortes de la siguiente forma: glicemia en ayunas de $105 \mathrm{mg} / \mathrm{dL}$, glicemia 1 hora post-ingesta de carga de glucosa de $190 \mathrm{mg} / \mathrm{dL}, 2$ horas después de $165 \mathrm{mg} / \mathrm{dL}$ y 3 horas después de 145 $\mathrm{mg} / \mathrm{dL}$. Por otro lado, Carpenter y Coustan definen los cortes de la siguiente forma: glicemia en ayunas de $95 \mathrm{mg} / \mathrm{dL}$, glicemia 1 hora post-ingesta de carga de glucosa de $180 \mathrm{mg} / \mathrm{dL}, 2$ horas después de $155 \mathrm{mg} / \mathrm{dL}$ y 3 horas después de $140 \mathrm{mg} / \mathrm{dL}$. (1,7). Si bien es cierto que con los valores establecidos por Carpenter y Coustan se darían una mayor cantidad de diagnósticos de DMG (de hasta un $50 \%$ basado en algunos estudios), otros estudios demuestran que aquellas mujeres que no son diagnosticadas debido al uso de valores mayores tienen mayores índices de complicaciones perinatales respecto a la población normal $(1,2)$. En ausencia de estudios comparativos claros, no se puede recomendar un set de valores diagnósticos sobre el otro. Se recomienda a los profesionales en salud seleccionar un sólo set diagnóstico para uso consistente con sus pacientes, basándose posiblemente en la prevalencia de diabetes en sus comunidades y la disposición de recursos para el tratamiento de la DMG de ser esta diagnosticada (2).

Se ha visto que las embarazadas con un solo valor anormal en la curva de tolerancia de glucosa, tienen un riesgo significativamente mayor en comparación a la población normal de efectos adversos perinatales. Se necesita de más estudios para determinar exactamente los riesgos que conlleva y los posibles beneficios que tendría la terapia en estas pacientes, sin embargo, la ACOG recientemente considera la posibilidad de utilizar un sólo valor alterado para realizar el diagnóstico de DMG y no dos como se realiza tradicionalmente (2).

\section{- DIAGNÓSTICO DE UN SOLO PASO}

A partir del 2010, la Asociación Internacional del Grupo de Estudio de Diabetes y Embarazo (IADPSG, por sus siglas en inglés) comenzó a promover el uso de un sólo paso diagnóstico con la prueba de tolerancia oral con 75 gramos de glucosa de 2 horas para el diagnóstico de la DMG a las 24-28 semanas de gestación. Con este método, se establecería el diagnóstico de haber al menos un valor igual o superior al establecido para las medidas (glicemia 
en ayunas $92 \mathrm{mg} / \mathrm{dL}, 1$ hora post ingesta $180 \mathrm{mg} / \mathrm{dL}$, o 2 horas post ingesta 153 $\mathrm{mg} / \mathrm{dL})(1,2)$.

En el 2011, la Asociación Americana de Diabetes (ADA por sus siglas en inglés), reconoce que con este método se incrementaría la prevalencia de DMG y posiblemente los gastos médicos. Otros factores negativos citados por la el $\mathrm{NIH}$ son la falta de estudios que demuestren los beneficios del uso de este método sobre el de dos pasos, y reafirma el aumento en costos médicos como una potencial consecuencia negativa. Además, destaca el hecho de que para la prueba de tamizaje con 50 gramos de glucosa no se requiere de ayuno, lo que posiblemente es más práctico para las usuarias. En el 2013, un reporte realizado con base en el consenso del Instituto Nacional Eunice Kennedy Shriver respecto al diagnóstico de la DMG, secunda la posición del $\mathrm{NIH}$, e indica que no hay evidencia que indique una mejoría clínica significativa de resultados obstétricos o maternos con el enfoque de un sólo paso $(1,2)$.

No obstante, un estudio de seguimiento demostró que las mujeres diagnosticadas gracias al uso del método de un paso presentaban un riesgo 3.4 veces mayor de desarrollar pre-diabetes y diabetes tipo 2, y tuvieron hijos con un riesgo aumentado de obesidad y sobrepeso, lo que sugiere que estas mujeres se podrían beneficiar de un seguimiento más estricto que acompaña el diagnóstico de DMG (8). Otro estudio refuerza lo anterior, al concluir que el uso del diagnóstico de un sólo paso es costoefectivo solamente en el caso de que las mujeres que presentaron DMG reciban consejo postparto y seguimiento para la prevención de la diabetes tipo 2 (1).
Para el 2015, un estudio Cochrane concluye que ninguna estrategia de tamizaje ha mostrado ser óptima (9). Aún para el 2019, la ADA reconoce que hay ausencia de evidencia clara que apoye este nuevo método diagnóstico, ya que faltan estudios que comparen un método diagnóstico contra el otro (1).

La ACOG por su parte, concluye que las mujeres que fueran diagnosticadas de esta forma podrían estar en menor riesgo de eventos adversos y podrían no beneficiarse del diagnóstico y tratamiento en comparación a las mujeres diagnosticadas por los criterios tradicionales, por lo que continúa apoyando el uso de los 2 pasos para el diagnóstico de la DMG y recomienda el estudio de las implicaciones de posibles cambios futuros antes de su aplicación a nivel mundial. Asimismo, se reconoce que las prácticas individuales e institucionales podrían funcionar en virtud a la población en práctica $(1,2)$.

\section{- TAMIZAJE TEMPRANO}

En ocasiones se dificulta diferenciar entre una diabetes mellitus gestacional y una pre-gestacional debido a que muchas mujeres no se realizan el tamizaje previo al embarazo (2). Es por esto que se recomienda realizar un tamizaje temprano, idealmente al inicio del cuidado prenatal con el fin de identificar a las mujeres con diabetes tipo 2 no diagnosticada, en todas aquellas mujeres con sobrepeso u obesidad y uno - más factores de riesgo, entre los cuales se citan: sedentarismo, familiar de primer grado con diabetes, alto riesgo por raza o etnia (afroamericanos, latinos, amerindio, asiáticos-americanos 0 isleños del Pacífico), infante previo con 
peso mayor a $4000 \mathrm{~g}$, diabetes mellitus gestacional previa, hipertenso 0 en terapia anti-hipertensiva, colesterol HDL menor a $35 \mathrm{mg} / \mathrm{dL}$ o triglicéridos mayores a $250 \mathrm{mg} / \mathrm{dL}$, síndrome de ovario poliquístico, $\mathrm{HbA1c}$ igual o mayor a $5.7 \%$, intolerancia a los carbohidratos o intolerancia en ayunas en pruebas realizadas anteriormente, historia de enfermedad cardiovascular $u$ otras condiciones que se asocien a resistencia de insulina tales como acantosis nigricans o IMC mayor a $40(1,6,10)$.

Aún no se ha determinado la mejor prueba de tamizaje para estos casos (11). El tamizaje para diagnosticar una diabetes mellitus tipo 2 en mujeres que no están embarazadas con una glicemia en ayunas seguida de una ingesta de 75 gramos de glucosa y una medición de 2 horas podría utilizarse con este propósito (12). Sin embargo, muchos obstetras utilizan el tamizaje de dos pasos que se realiza normalmente para el diagnóstico de DMG (2).

Otros estudios revelan que cuando los niveles de hiperglicemia inicialmente detectados en cualquier momento del embarazo coinciden $o$ superan los niveles determinados para el diagnóstico de mujeres diabéticas no embarazadas (glucosa en ayunas mayor o igual a 126 $\mathrm{mg} / \mathrm{dL}$, prueba de tolerancia con carga de 75 gramos de 2 horas mayor o igual a $200 \mathrm{mg} / \mathrm{dL}$ o una glucosa al azar mayor o igual a $200 \mathrm{mg} / \mathrm{dL}$ asociada a síntomas de diabetes), se considerará como diabetes en el embarazo o pregestacional. Es más común que la diabetes pre-gestacional ocurra en el primer trimestre de embarazo, a diferencia de con la DMG, que también puede ocurrir en cualquier momento del embarazo pero predominantemente luego de las 24 semanas (4).

La ADA indica que se puede utilizar la medición de hemoglobina glicosilada para el diagnóstico, pero que probablemente no sea un buen parámetro para utilizar de forma exclusiva, ya que tiene una sensibilidad disminuida en comparación al uso de pruebas de tolerancia de glucosa $(1,13)$.

El tamizaje a las 24-28 semanas para DMG es recomendado aún cuando el tamizaje temprano en el embarazo haya sido negativo. En mujeres cuyo tamizaje haya resultado positivo temprano en el embarazo, pero con pruebas diagnósticas negativas, se pueden utilizar las pruebas diagnósticas directamente sin volver a realizar el tamizaje a las 24-28 semanas (2).

\section{MANEJO Y TRATAMIENTO}

La DMG constituye un factor de riesgo para el desarrollo de diversas patologías. Entre estas cabe mencionar preeclampsia, cesárea, desarrollo de diabetes mellitus tipo 2, macrosomía, distocia de hombro, hipoglicemia fetal, hiperbilirrubinemia, trauma relacionado al nacimiento y óbitos (2). Es entonces importante realizar un manejo adecuado de la diabetes para así lograr disminuir estos riesgos. De acuerdo con el último boletín de la ACOG para el tratamiento de la diabetes en el embarazo, al instaurar el tratamiento se ha observado una disminución en las complicaciones previamente mencionadas, y a su vez se ha visto disminución de infantes grandes para edad gestacional (2).

Tanto la ADA como la ACOG, NICE y FIGO dividen el manejo de la DMG en 
cambios en el estilo de vida y tratamiento farmacológico.De acuerdo con la ACOG, ADA y FIGO, el tratamiento inicial de estas pacientes consiste en la instauración de cambios en el estilo de vida, sin que exista un valor umbral para la instauración de tratamiento farmacológico $(1,2,4)$.

Por su parte, la guía NICE indica que es necesaria la valoración de los niveles de glucosa, siendo entonces que si la paciente tiene glicemia en ayunas de $7 \mathrm{mmol} / \mathrm{L}(126 \mathrm{mg} / \mathrm{dL})$ o menos se puede realizar manejo con cambios en estilo de vida. Sin embargo, si los niveles son superiores se debe valorar el tratamiento conjunto.

En casos en los que no se logre control glicémico o en los que la glicemia en ayunas sea igual o superior a $126 \mathrm{mg} / \mathrm{dL}$ se sugiere el uso de fármacos aunado a cambios dietéticos y actividad física (14).

En un estudio realizado en 2017 se intentaron encontrar predictores para el tratamiento de la diabetes, se analizaron múltiples variables y se concluyó que el único factor predictivo para la necesidad de tratamiento farmacológico con metformina o metformina e insulina fue el IMC. Además, indican que esto es consistente con lo reportado en otros estudios en los cuales se ha visto que otras variables como IMC, edad, etnia, paridad y DMG previa, podrían afectar el control glicémico (15).

\section{- ACTIVIDAD FÍSICA}

Se recomienda actividad física en las pacientes con DMG con el objetivo de mejorar el control glicémico. Se aconseja actividad aeróbica de moderada intensidad por 30 minutos al menos 5 días a la semana. (2). Se aconseja adaptar la actividad física individualmente, pudiendo mantener la actividad física previa al embarazo en caso de las pacientes físicamente activas - realizar caminatas o ejercicios de brazos por 10 minutos luego de cada comida (4).

\section{- TERAPIA MÉdICA DE NUTRICIÓN}

Existen pocos estudios bien elaborados que respalden la forma adecuada de implementarla. En 2017 se realizó un metaanálisis en el cual se comparan 19 estudios. En el mismo no se logran evidenciar diferencias significativas en desenlaces (hipertensión, grande para edad gestacional, mortalidad perinatal, desarrollo de diabetes mellitus tipo 2 y mortalidad 0 morbilidad neonatal) al implementar cambios dietéticos en mujeres con diagnóstico de DMG. Es importante aclarar que en dicho artículo se encontró que muchos de los artículos utilizados aportan evidencia de baja calidad (16). Pese a lo anterior, la terapia médica de nutrición constituye la primera línea tratamiento. Las guías de manejo sugieren que el plan nutricional debe ser realizado por un nutricionista (1). Además, se resalta la importancia de brindar educación nutricional enfocada en la reducción, moderación y métodos de cocción (4). El objetivo de esta terapia consiste en alcanzar normoglicemia en ausencia de cetosis considerando un aporte calórico y nutricional que lleve a una ganancia de peso adecuada y desarrollo fetal óptimo $(2,4,17)$.

\section{- CARBOHIDRATOS}

Se ha considerado la disminución del contenido de carbohidratos en la dieta 
como parte del abordaje a seguir (17). Se sugiere limitar el consumo de carbohidratos a $33-40 \%$ de las calorías consumidas, con un consumo de al menos $175 \mathrm{~g}$ de carbohidratos por día $(1,2)$. Esto con el fin de disminuir el riesgo de macrosomía, ya que se ha visto que los carbohidratos tienen efecto sobre las concentraciones glicémicas postprandiales, las cuales se han relacionado con la aparición de la misma (17). Cabe destacar que pese a esta recomendación, existe controversia en cuanto al efecto que se podría observar. Se habla de que una disminución en el consumo de carbohidratos podría ocasionar un aumento del consumo de grasas, lo que podría exacerbar la resistencia a la insulina. $(17,18)$.

Se ha observado que el consumo de carbohidratos complejos, cuya absorción es más lenta, podría ser más efectivo en el control glicémico que la restricción de los mismos, logrando así una disminución del riesgo de hiperglicemia postprandial y la resistencia a insulina $(2,18)$.

\section{- FIBRA}

Se recomienda un consumo de un máximo de $28 \mathrm{~g}$ de fibra (en especial soluble) diarios, con el fin de disminuir los lípidos séricos y limitar los picos glicémicos (4).

\section{- PROTEÍNAS Y GRASAS}

La ADA recomienda un mínimo de $71 \mathrm{~g}$ de proteína al día, mientras que la ACOG indica que estas deben corresponder al $20 \%$ de las calorías diarias $(1,2)$. Se ha visto que un consumo prenatal proteico elevado resulta en una disminución del peso al nacer y que puede ser más perjudicial que el bajo consumo proteico. Es por tanto que se plantea un consumo proteico moderado en el embarazo (17).

Por su parte, el consumo de grasas se debe limitar a $40 \%$ del total de calorías diarias (2). En un estudio realizado en China se logró comprobar que un aumento en el consumo de ácidos grasos poliinsaturadas puede beneficiar a la madre y al feto. Se observó una disminución de la glicemia en ayunas, la glicemia 2 horas postprandial y el índice de sensibilidad a insulina aumentó, sin evidenciar un aumento significativo en niveles de lípidos al comparar con el grupo control (19).

\section{- ÍNDICE GLICÉMICO}

Los datos que apoyan la recomendación del consumo de alimentos con bajo índice glicémico se extraen de estudios realizados en mujeres no embarazadas, en los que se ha visto que los alimentos con índice bajo llevan a una reducción de $0,4 \%$ en la hemoglobina glicosilada, y en mujeres no diabéticas embarazadas se ha visto que la dieta basada en este tipo de alimentos lleva a una disminución del peso al nacer (4). Sin embargo, en un análisis sistemático se vio que al comparar dietas de alto y bajo índice glicémico en mujeres con DMG no se observan diferencias significativas en desenlaces como grande para edad gestacional, cesáreas, hipertensión o eclampsia (16). En un estudio realizado con 63 mujeres se comparó la necesidad de insulina luego de la instauración de dieta de bajo índice glicémico versus dieta convencional, alta en fibra y con mayor índice glicémico. Se observó que una mayor cantidad de pacientes en el 
segundo grupo mencionado requirieron tratamiento con insulina (17).

\section{TRATAMIENTO FARMACOLÓGICO}

\section{- HIPOGLICEMIANTES ORALES}

De acuerdo con la ADA el tratamiento farmacológico de elección para el manejo de la DMG es la insulina (1). Sin embargo, y pese a no estar aprobadas por la FDA, la metformina y gliburida son utilizadas para el tratamiento de la DMG (2). La sociedad para la medicina materno fetal (20), en su enunciación del 2018, concluye que en mujeres en las que no se logra control adecuado con terapia médica nutricional se puede utilizar de forma segura la metformina como primera línea de tratamiento (20). Esta recomendación también se puede encontrar en las guías NICE (14).

La metformina es una biguanida que actúa inhibiendo la gluconeogénesis hepática, aumentando la captación de glucosa por tejidos periféricos, además de que disminuye la absorción gastrointestinal de glucosa (2). Sin embargo, la misma cruza la placenta, y se ha observado que las concentraciones fetales son similares a las de la madre (20).

Una publicación realizada en 2018 en respuesta a la declaración de la SMFM, menciona que los efectos a largo plazo de fetos expuestos a metformina in útero no son del todo conocidos. Posterior a la declaración de la SMFM se publicaron los resultados de 2 estudios en los que evidencian el posible aumento del riesgo de obesidad infantil en niños expuestos a metformina intraútero. También se ha visto que la exposición a este fármaco podría aumentar el riesgo de hígado graso no alcohólico en niños (21).

La metformina tiene efectos antiangiogénicos, lo que puede llevar a la reducción del riesgo de preeclampsia (21). El uso de metformina comparado con el uso de insulina también se ha relacionado con menor aumento de peso materno, menor edad gestacional al nacer y menos hipoglicemia neonatal $(4,20)$. Pese a esto, también se ha visto un aumento en el número de partos pretérmino al utilizar metformina (21)

Se recomienda una dosis inicial de $500 \mathrm{mg}$ en la noche. Si fuese necesario se podría aumentar a $500 \mathrm{mg}$ dos veces al día, con dosis máximas de 2500$3000 \mathrm{mg}$ divididos en 2 o 3 dosis al día (2). La gliburida, una sulfonilurea, actúa aumentado la secreción pancreática de insulina. Se requieren dosis divididas de 2.5-20mg, y en algunos casos podrían ser necesarios $30 \mathrm{mg}$ por día (2). Al utilizarla las concentraciones fetales pueden alcanzar hasta el $70 \%$ de las concentraciones maternas (20). Se ha evidenciado, en revisiones sistemáticas y estudios observacionales, que al comparar gliburida e insulina, el uso de gliburida resulta en pesos mayores al nacer, 2 veces más riesgo de hipoglicemia neonatal y macrosomía, más casos de preeclampsia, óbitos e hiperbilirrubinemia (4).

Estudios han demostrado que $4-16 \%$ de las pacientes tratadas inicialmente con gliburida requieren incorporar insulina al régimen de tratamiento para lograr el control glicémico (2). En un estudio presentado en el American Journal of Obstetrics and Gynecology, se intentó identificar a las pacientes en las que el tratamiento con gliburida fallaría. Se logró identificar estas pacientes desde el 
inicio del tratamiento al incluir factores como DMG previa, edad gestacional al diagnóstico $\leq 26$ semanas, $\geq 7$ glicemias postprandiales mayores a $120 \mathrm{mg} / \mathrm{dL}$ en la semana de inicio del tratamiento, $\geq 1$ glicemia mayor a $200 \mathrm{mg} / \mathrm{dL}$ en la semana del inicio del tratamiento, prueba de sobrecarga oral de glucosa $\geq 228 \mathrm{mg} / \mathrm{dL}$ y valor a la hora de la curva de tolerancia a glucosa $\geq 221 \mathrm{mg} / \mathrm{dL}$ (22).

Debido a todo lo anterior, la ACOG no recomienda la gliburida como primera opción de tratamiento (2).

\section{- INSULINA}

Esta opción terapéutica no cruza la placenta. Dentro de los tipos de insulina existen los análogos de acción rápida (insulinas lispro y aspart), las de acción corta (insulina regular), acción intermedia (NPH) y acción prolongada (insulina glargina y detemir) (1). Se debe considerar la insulina como primera línea en pacientes con diagnóstico de DMG antes de las 20 semanas de gestación, necesidad para terapia farmacológica luego de las 30 semanas, glicemia en ayunas mayor a $110 \mathrm{mg} / \mathrm{dL}$, glicemia 1hora postprandial mayor a $140 \mathrm{mg} / \mathrm{dL}$ y ganancia de peso mayor a $12 \mathrm{~kg}$ (4).

Se recomienda una dosis diaria inicial de $0.7-1.0 \mathrm{U} / \mathrm{kg}$ la cual se puede dividir de acuerdo a los requerimientos de la paciente. Esto ya que el tratamiento se enfoca en evitar hiperglicemias, por cuanto se debe tener registro de las glicemias de la paciente y así lograr orientar adecuadamente el tratamiento (2). Se sabe que los requerimientos basales de insulina de la paciente embarazada aumentan al avanzar el embarazo, por lo que puede ser necesario realizar ajustes siendo en primer trimestre necesaria una dosis de $0.7 \mathrm{U} / \mathrm{kg} /$ día, $0.8 \mathrm{U} / \mathrm{kg} /$ día a partir de la semana 18, 0.9U/ $/ \mathrm{kg} /$ día desde la semana 26 hasta llegar a 1U/kg/día desde la semana 36 (4). En las semanas 9 a 16 podría existir una disminución en los requerimientos de insulina, por lo que se podrían dar hipoglicemias (1). Cabe destacar que esto puede variar, siendo necesario individualizar el tratamiento.

Para lograr simular la secreción fisiológica, se aconseja el uso de insulina de acción rápida e insulina de acción intermedia o larga duración. Es por esto que se ha utilizado el régimen de insulina regular con NPH. Pese a esto, se ha visto que la insulina regular no logra controlar las hiperglicemias postprandiales; y con el uso de insulina $\mathrm{NPH}$, debido a su acción en mujeres embarazadas, se podrían requerir hasta 3 dosis diarias, lo que podría ocasionar hipoglicemias (4).

Con base en lo anterior, sería posible utilizar análogos para también reproducir de forma más efectiva la curva fisiológica. La insulina aspart ha sido comparada con insulina regular, y se ha visto que ofrece mejor control glicémico; además, la incidencia de efectos adversos y malformaciones congénitas es similar (4). En cuanto a las insulinas de acción prolongada, no se han identificado problemas de seguridad al utilizar insulina detemir, sin embargo, las investigaciones se han concentrado en pacientes con diabetes tipo 1 (4). Al comparar el uso de detemir con NPH en pacientes con diabetes tipo 1 , se ha observado que la primera podría reducir de forma más efectiva las glicemias en ayunas con desenlaces maternos $y$ fetales similares a NPH (23). La glargina 
es otra opción terapéutica, que al compararla con $\mathrm{NPH}$ se han visto desenlaces maternos y fetales similares, sin reporte de efectos adversos, sin embargo, los estudios con los que se cuenta son más limitados (23).

\section{MONITOREO DEL TRATAMIENTO}

Una vez instauradas las medidas de cambios en el estilo de vida, se recomienda un espacio de 1-2 semanas para revalorar la evolución de la paciente y tomar decisiones de manejo (14). Tanto la ACOG como la ADA recomiendan evaluar a la paciente cada semana posterior a la instauración del tratamiento. Como parámetros para el monitoreo sugieren como meta valores de $95 \mathrm{mg} / \mathrm{dL}$ para glicemia en ayunas, $140 \mathrm{mg} / \mathrm{dL}$ para la glicemia 1hora postprandial o $120 \mathrm{mg} / \mathrm{dL}$ dos hora postprandial; si estos no son alcanzados, se debe adicionar nuevo tratamiento o modificación de la dosis $(1,2)$.

En el 2018, se publica un artículo el cual busca idear un sistema de puntuación para guiar el tratamiento de las pacientes con DMG. Se utilizaron como parámetros la glicemia en ayunas, la glicemia postprandial, la circunferencia abdominal fetal, el IMC y la edad gestacional. Posteriormente se asignaron valores de 2 a 2 para cada uno dependiendo del resultado obtenido al evaluar a la paciente para luego realizar una sumatoria. Si la misma obtuvo una puntuación total menor a 0 , recomendaron realizar una nueva consulta con nutricionista, y si la puntuación se encontraba entre 0 y 1 se debe mantener dieta y actividad física. Por su parte, para las pacientes que tuvieron puntuación de 2 a 4 se recomendó el inicio de terapia farmacológica oral, y en las que se obtuvo una calificación mayor a 4 se recomendó tratamiento con insulina $y$ dieta con o sin hipoglicemientes orales. Los autores concluyen que el sistema es útil como herramienta adjunta a la experiencia médica, y que la misma no afectó negativamente la toma de decisiones respecto al tratamiento ni tuvo efecto adverso en los desenlaces perinatales (24).

\section{MOMENTO DEL PARTO Y MANEJO}

Al existir menos evidencia respecto al momento del parto y la vigilancia fetal en las pacientes con DMG, se recomienda iniciar monitoreos fetales semanales a partir de la semana 34-36 de embarazo en las pacientes con pobre control glicémico (23).

Un estudio realizado de forma retrospectiva, si bien con confusores no ajustados como obesidad materna, evidenció que existe un aumento del riesgo de óbitos a las 36-39 semanas, con un aumento significativo en la semana 39 y 40 al comparar el manejo expectante con la inducción del parto (23). Sin embargo, según la ACOG, en casos de buen control glicémico que reciben tratamiento médico, no es necesario el parto antes de las 39 semanas (2). Otro factor a tomar en cuenta a la hora de decidir el momento del parto es el tamaño fetal. En un estudio publicado en 2015, se dividieron pacientes con embarazos únicos y fetos por encima del percentil 95 en dos grupos de forma aleatoria, 409 pacientes recibirían inducción del parto entre las semanas $37-38+6$, mientras que 413 recibirían manejo expectante. Cabe 
destacar que no sólo había pacientes con DMG. Se observó mayor probabilidad de parto vaginal espontáneo en el grupo de inducción, además hubo menor riesgo de distocia de hombros en este grupo. Pese a esto, se observaron mayor cantidad de casos de hiperbilirrubinemia en el grupo de inducción. No se vio diferencia significativa en cuanto a cesáreas entre los grupos (25).

En el caso de pacientes que no logren el control glicémico la ACOG indica que el parto entre la semana $37-38+6$ puede estar justificado y se debe reservar el mismo en pacientes con edad gestacional de 34-36+6 que no logren la meta glicémica aún estando hospitalizadas (2).

El manejo glicémico del parto tiene como objetivo prevenir la hiperglicemia e hipoglicemia neonatal sin ocasionar hipoglicemia materna. Se sabe que los requerimientos de insulina disminuyen durante el parto (23). Se ha recomendado una meta glicémica de $72 \mathrm{mg} / \mathrm{dL}$ a $126 \mathrm{mg} / \mathrm{dL}$ durante el parto, por lo que se deben realizar controles capilares cada hora. En los casos en los que no se logre esta meta se puede utilizar infusión de dextrosa e insulina (14). Sin embargo, existen pocos estudios que respalden el uso de un protocolo específico de manejo intraparto. Se recomienda que cada centro de salud tenga sus propios protocolos de manejo, los cuales detallen monitoreo y régimen de tratamiento (23).

\section{CONCLUSIONES}

Con esta revisión bibliográfica se logra concluir que al ser la diabetes mellitus gestacional una patología tan importante, con una prevalencia que va en aumento $y$ efectos negativos a nivel de la comunidad, se debe recalcar la importancia de unificar los lineamientos a seguir en cuanto a su diagnóstico y tratamiento. Cualquier grupo de criterios diagnósticos de DMG propuestos, necesitará evolucionar a partir de un consenso, en el cual se tomen en cuenta los riesgos y beneficios de cada método y las implicaciones sociales, económicas y clínicas. Asimismo, queda mucho por explorar y definir a nivel de tratamiento, específicamente en cuanto a recomendaciones no farmacológicas y efectos adversos a largo plazo del tratamiento farmacológico. El diagnóstico temprano, el tratamiento adecuado y el seguimiento cercano de esta patología, son pilares importantes a la hora de disminuir las complicaciones asociadas a la misma y con el fin de tener resultados exitosos.

\section{REFERENCIAS}

1. American Diabetes Association. Classification and Diagnosis of Diabetes: Standards of Medical Care in Diabetes 2019. Diabetes Care. 2018 Dec 17;42(Supplement 1):S13-S28. https://doi.org/10.2337/dc19$\underline{\mathrm{s} 002}$

2. ACOG Practice Bulletin No. 190. Obstetrics \& Gynecology. 2018 02;131(2):e49-e64. https://doi.org/10.1097/aog.0000000000002501 
3. Bouthoorn SH, Silva LM, Murray SE, Steegers EAP, Jaddoe VWV, Moll H, Hofman A, Mackenbach JP, Raat H. Low-educated women have an increased risk of gestational diabetes mellitus: the Generation $\mathrm{R}$ Study. Acta Diabetologica. 2014 Oct 26;52(3):445-452. https://doi.org/10.1007/s00592-014-0668-x

4. Hod M, Kapur A, Sacks DA, Hadar E, Agarwal M, Di Renzo GC, Roura LC, Mclntyre HD, Morris JL, Divakar H. The International Federation of Gynecology and Obstetrics (FIGO) Initiative on gestational diabetes mellitus: A pragmatic guide for diagnosis, management, and care\#. International Journal of Gynecology \& Obstetrics. 2015 Oct;131:S173-S211. https://doi.org/10.1016/s0020-7292(15)30033-3

5. Gabbe SG, Niebyl JR, Simpson JL, Landon MB, Galan HL, Jauniaux ER, Driscoll DA, Berghella V, Grobman WA. Diabetes Mellitus Complicating Pregnancy. En: Landon M, Catalano P, Gabbe S. Obstetrics Normal and Problem Pregnancies. Seventh Edition. Philadelphia: Elsevier; 2017. p. 862-899.

6. Moyer VA. Screening for Gestational Diabetes Mellitus: U.S. Preventive Services Task Force Recommendation Statement. Annals of Internal Medicine. 201403 18;160(6):414-420. https://doi.org/10.7326/m13-2905

7. American Diabetes Association. Management of Diabetes in Pregnancy: Standards of Medical Care in Diabetes-2019. Diabetes Care. 2018 Dec 17;42(Supplement 1):S165-S172. https://doi.org/10.2337/dc19-s014

8. Lowe WL, Scholtens DM, Lowe LP, Kuang A, Nodzenski M, Talbot O, Catalano PM, Linder B, Brickman WJ, Clayton P, Deerochanawong C, Hamilton J, Josefson JL, Lashley M, Lawrence JM, Lebenthal Y, Ma R, Maresh M, McCance D, Tam WH, Sacks DA, Dyer AR, Metzger BE HAPO Follow-up Study Cooperative Research Group. Association of Gestational Diabetes With Maternal Disorders of Glucose Metabolism and Childhood Adiposity. JAMA. 201809 11;320(10):1005-1016. https://doi.org/10.1001/jama.2018.11628

9. Farrar D, Duley L, Medley N, Lawlor DA. Different strategies for diagnosing gestational diabetes to improve maternal and infant health. Cochrane Database of Systematic Reviews. 2015; 1. Art. No.: CD007122. (Meta-analysis). https://doi.org/ 10.1002/14651858.CD007122.pub3

10. Poltavskiy E, Kim DJ, Bang H. Comparison of screening scores for diabetes and prediabetes. Diabetes Research and Clinical Practice. 2016 08;118:146-153. https://doi.org/10.1016/j.diabres.2016.06.022

11. McIntyre HD, Sacks DA, Barbour LA, Feig DS, Catalano PM, Damm P, McElduff A. Issues with the diagnosis and classification of hyperglycemia in early pregnancy. Diabetes Care. 2015 Oct 30;39(1):53-54. https://doi.org/10.2337/dc15-1887

12. Chamberlain JJ, Rhinehart AS, Shaefer CF, Neuman A. Diagnosis and Management of Diabetes: Synopsis of the 2016 American Diabetes Association Standards of Medical Care in Diabetes. Annals of Internal Medicine. 201603 01;164(8):542-552. https://doi.org/10.7326/m15-3016

13. Khalafallah A, Phuah E, Al-Barazan AM, Nikakis I, Radford A, Clarkson W, Trevett C, Brain T, Gebski V, Corbould A. Glycosylated haemoglobin for screening and diagnosis of gestational diabetes mellitus. BMJ Open. 2016 04;6(4):e011059. https://doi.org/10.1136/bmjopen-2016-011059

14. NICE guideline. Diabetes in pregnancy: management from preconception to the postnatal period [Internet]. The National Institute for Health and Care Excellence. 2019 [cited 9 February 2019]. Available from: https://www.nice.org.uk/guidance/ng3 
15. Ali A, Shastry S, Nithiyananthan R, Ali A, Ganapathy R. Gestational diabetes-Predictors of response to treatment and obstetric outcome. European Journal of Obstetrics \& Gynecology and Reproductive Biology. 2018 01;220:57-60. https://doi.org/10.1016/j.ejogrb.2017.11.014

16. Han S, Middleton P, Shepherd E, Van Ryswyk E, Crowther CA. Different types of dietary advice for women with gestational diabetes mellitus. . Cochrane Database of Systematic Reviews. 2017;2: CD009275. https://doi.org/10.1002/14651858.cd009275.pub3

17. Thomas A, Duarte-Gardea M. Nutritional Management for Gestational Diabetes. Nutrition in the Prevention and Treatment of Disease. 4th ed. 2017. p. 709-729. https://doi.org/10.1016/B978-0-12802928-2.00032-1

18. Hernandez TL, Brand-Miller JC. Nutrition Therapy in Gestational Diabetes Mellitus: Time to Move Forward. Diabetes Care. 201806 22;41(7):1343-1345. https://doi.org/10.2337/dci18-0014

19. Wang $\mathrm{H}$, Jiang $\mathrm{H}$, Yang $\mathrm{L}$, Zhang $\mathrm{M}$. Impacts of dietary fat changes on pregnant women with gestational diabetes mellitus: a randomized controlled study. Asia Pac J Clin Nutr. 2015;24(1):58-64. https://doi.org/10.6133/apjcn.2015.24.1.19

20. SMFM Statement: Pharmacological treatment of gestational diabetes. American Journal of Obstetrics and Gynecology. 2018 05;218(5):B2-B4. https://doi.org/10.1016/i.ajog.2018.01.041

21. Barbour LA, Scifres C, Valent AM, Friedman JE, Buchanan TA, Coustan D, Aagaard K, Thornburg KL, Catalano PM, Galan HL, Hay WW, Frias AE, Shankar K, Simmons RA, Moses RG, Sacks DA, Loeken MR. A cautionary response to SMFM statement: pharmacological treatment of gestational diabetes. American Journal of Obstetrics and Gynecology. 2018 Oct;219(4):367.e1-367.e7. https://doi.org/10.1016/i.ajog.2018.06.013

22. Harper LM, Glover AV, Biggio JR, Tita A. Predicting failure of glyburide therapy in gestational diabetes. Journal of Perinatology. 201601 21;36(5):347-351. https://doi.org/10.1038/jp.2015.216

23. Feig DS, Berger H, Donovan L, Godbout A, Kader T, Keely E, Sanghera R. Diabetes and Pregnancy. Canadian Journal of Diabetes. 2018 04;42:S255-S282. https://doi.org/10.1016/i.jcjd.2017.10.038

24. Do Valle JB, Silva JC, Oliveira DS, Martins L, Lewandowski A, Horst W. Use of a clinical-laboratory score to guide treatment of gestational diabetes. International Journal of Gynecology \& Obstetrics. 2017 Oct 11;140(1):47-52. https://doi.org/10.1002/iigo.12326

25. Boulvain M, Senat M, Perrotin F, Winer N, Beucher G, Subtil D, Bretelle F, Azria E, Hejaiej D, Vendittelli F, Capelle M, Langer B, Matis R, Connan L, Gillard P, Kirkpatrick C, Ceysens G, Faron G, Irion O, Rozenberg P. Induction of labour versus expectant management for large-for-date fetuses: a randomised controlled trial. The Lancet. 2015 06;385(9987):2600-2605. https://doi.org/10.1016/s0140-6736(14)61904$\underline{8}$ 